\title{
miR-23b and miR-27b are oncogenic microRNAs in breast cancer: evidence from a CRISPR/Cas9 deletion study
}

\author{
Bethany N. Hannafon, Angela Cai, Cameron L. Calloway, Yi-Fan Xu, Roy Zhang, Kar-Ming Fung and Wei-Qun Ding ${ }^{*}$ (D)
}

\begin{abstract}
Background: Altered expression of microRNAs (miRNAs) is known to contribute to cancer progression. miR-23b and miR-27b, encoded within the same miRNA cluster, are reported to have both tumor suppressive and oncogenic activity across human cancers, including breast cancer.

Methods: To clarify this dichotomous role in breast cancer, miR-23b and miR-27b were knocked out using CRISPR/ Cas9 gene knockout technology, and the role of endogenous miR-23b and miR-27b was examined in a breast cancer model system in vitro and in vivo.

Results: Characterization of the knockout cells in vitro demonstrated that miR-23b and miR-27b are indeed oncogenic miRNAs in MCF7 breast cancer cells. miR-23b and miR-27b knockout reduced tumor growth in xenograft nude mice fed a standard diet, supporting their oncogenic role in vivo. However, when xenograft mice were provided a fish-oil diet, miR-27b depletion, but not miR-23b depletion, compromised fish-oil-induced suppression of xenograft growth, indicating a context-dependent nature of miR-27b oncogenic activity.
\end{abstract}

Conclusions: Our results demonstrate that miR-23b and miR-27b are primarily oncogenic in MCF7 breast cancer cells and that miR-27b may have tumor suppressive activity under certain circumstances.

Keywords: CRISPR/Cas9, microRNA, Breast cancer

\section{Background}

miRNAs are small, non-protein coding RNAs, ranging in sizes from 17 - to 25 nucleotides that regulate mRNA expression at the post-transcriptional level by complementary binding to corresponding sequences in the 3' untranslated region (3'UTR). miRNAs are predicted to regulate $>90 \%$ of protein-coding genes, thereby making them the largest class of gene regulators [1]. Due to their broad regulatory role and their various target mRNAs, miRNAs can regulate various normal and pathogenic cellular processes including cell cycle, tumorigenesis, migration/invasion, and angiogenesis thus functioning as oncogenes or tumor suppressors [2].

The miR-23-27-24 family consists of two paralogs with the miR-23a cluster (miR-23a-27a-24-2) found on chromosome 19 and the intragenic miR-23b cluster (miR-23b-

\footnotetext{
* Correspondence: weiqun-ding@ouhsc.edu

Department of Pathology, University of Oklahoma Health Sciences Center, 940 Stanton L. Young Blvd., BMSB401A, Oklahoma City, OK 73104, USA
}

27b-24-1) located on chromosome 9 within the C9orf3 gene [3]. miR-23a and miR-27a differ from their paralogs by only one nucleotide near their 3 ' end, however, diverse expression patterns are observed among these two clusters [4] and both are predicted to have largely identical targets, although less than $15 \%$ of the same targets have been validated according to miRTarBase [5].

Based on the current literature, miR-23b and miR-27b have a dichotomous role in cancer progression. miR-23b expression is down-regulated in human glioma, prostate, bladder, breast, and gastrointestinal cancer, and has been shown to suppress tumor growth, invasion, angiogenesis, and metastasis, and influence chemo-resistance and tumor cell dormancy [6-15]. While other studies have shown that expression of miR-23b is elevated in various cancers and may also function as an oncogene by promoting tumor growth, proliferation, and metastasis in glioma, prostate, breast, and gastric cancers and is associated with poor progression-free survival in 
ovarian cancer [16-20]. Likewise, expression of miR$27 \mathrm{~b}$ is increased in glioma, lymphoma, cervical, breast cancer, and acts as an oncogene by promoting proliferation, inhibiting apoptosis, and inducing migration and invasion [18, 21-23]. While other studies have shown that expression of miR-27b is reduced in lung, prostate, colorectal, gastric, and bladder cancer and it acts as a tumor suppressor by limiting proliferation, inhibiting tumor progression and angiogenesis, and the loss of miR-27b expression promotes epithelial to mesenchymal transition and breast cancer metastasis [9, 24-28]. Also, miR-23b and miR-27b have been shown to both promote $[29,30]$ and repress angiogenesis [31-33].

Studies examining the role of miR-23b and miR-27b in breast cancer have also demonstrated contrasting results, oncogenic or tumor suppressive. Several groups have demonstrated the cancer-promoting role of miR$23 \mathrm{~b}$ and miR-27b in breast cancer. For example, Jin et al., showed that inhibition of miR-23b and miR-27b by stable expression of anti-miR-23b/27b constructs reduces cell proliferation, anchorage-independent growth, migration, and invasion by metastatic breast cancer cell lines (MDA-MB-231-4175) in vitro and reduced tumor growth and metastases in vivo, in part by negatively regulating the target tumor suppressor gene, Nichscharin [18]. Likewise, Ell et al. demonstrated that miR-23b/27b expression is increased in three isogenic breast cancer progression cell line series (mouse $4 \mathrm{~T} 1$ series, and human MCF10A and MDA-MB-231 series). In this study, overexpression of the miR-23b-miR-27b-24 cluster in weakly metastatic 4TO7 cells led to an increase in the number of metastatic lung lesions in vivo in a tail-vein injection mouse model [34]. Wang et al. showed that inhibition of miR-27b using antagomirs in highly invasive breast cancer cells (MDA-MB-231-4175) suppresses cell invasion via upregulation of suppressor of tumorigenicity 14 (ST14), meanwhile, miR-27b overexpression stimulated invasion in moderately invasive breast cancer cells (ZR751) [23]. In contrast, other studies have shown a cancer-inhibitory effect of miR-23b and miR-27b in breast cancer. In a study by Pellegrino et al., miR-23b inhibition using a miRNA sponge construct leads to an increase in cell migration and metastatic spread in vivo, by directly inhibiting transcripts involved in cytoskeletal remodeling and motility in metastatic breast cancer cells (MDA-MB-231) [10]. Another study showed that down-regulation of miR-27b could confer tamoxifen resistance through up-regulation of NR5A2 and CREB1 expression in breast cancer cell lines (MCF7 and T47D), while upregulation of miR-27b expression enhanced the sensitivity of breast cancer cells to tamoxifen, suggesting a role for miR-27b in hormone therapy for breast cancer patients [35].
The observations that miR-23b and miR-27b act as either oncogenes or tumor suppressors in various cancer models are possibly due to the use of different model systems among various studies, but also likely due to the fact that these previous studies are unable to completely eliminate expression of miR-23b or miR-27b to elucidate their functional role in cancer. Because of their potential relevance in the development of anti-cancer therapeutics [36], we sought to determine the specific role of miR$23 \mathrm{~b}$ and miR-27b in breast cancer progression by genetic depletion of these miRNAs using CRISPR/Cas9 engineering technology. We developed miR-23b and miR-27b null breast cancer cell lines and characterized their phenotype in vitro using various phenotypic assays and in vivo using a xenograft mouse model. Our results indicate that miR-23b and miR-27b are primarily oncogenic in breast cancer cells.

\section{Methods \\ Cell culture}

We purchased the human breast cancer cell line MCF7 from the American Type Culture Collection (Manassas, VA). Upon receipt of the cells, low passage number aliquots were prepared and cryopreserved, and all experiments were conducted with low passage number cells. We have regularly checked the line for mycoplasma contamination by a PCR-based method and only mycoplasma free cells were used for this study. The cell line was genetically authenticated to be MCF7 by STR analysis at the Arizona University Genetic Core in 2018. The cells were cultured in DMEM containing $10 \%$ fetal bovine serum (FBS), $100 \mathrm{IU} / \mathrm{ml}$ penicillin and $100 \mu \mathrm{g} / \mathrm{ml}$ streptomycin (Corning/ Mediatech, Inc. Manassas, VA), at $37^{\circ} \mathrm{C}, 5 \% \mathrm{CO}_{2}$ in a humid environment. Media supplemented with 10\% FBS contains approximately $0.44 \% \mathrm{n}-3$ polyunsaturated fatty acids [37] and that total free fatty acid concentration in the fetal calf serum is around $200 \mu \mathrm{M}$, of which DHA account for about 3.9\% (below $10 \mu \mathrm{M}$ ) [38].

\section{Single guide RNA design and lentiviral transduction}

The sgRNAs were designed as recently described [39]. The DNA sequence corresponding to the annotated stemloop miRNA (miRBase) was used as an input sequence. The guides with the highest scores and/or closest proximity to the mature miRNA sequence were chosen. Oligonucleotides complimentary to the selected sgRNA sequence were synthesized with $B s m B I$ overhangs (Integrated DNA Technologies). The oligonucleotides were ligated into the lentiCRISPR v2, a gift from Feng Zhang (Addgene, \# 52961) [40]. miR-23b-sgRNA-1: 5'-CGTG GTTGCGTGGTAATCCC-3'; miR-23b-sgRNA-2: 5' GCTCTGGCTGCTTGGGTTCC-3'; miR-27b-sgRNA-1: 5'-GGTTTCCGCTTTGTTCACAG-3'; and miR-27b- 
sgRNA-2: 5' -AGGTGCAGAGCTTAGCTGAT-3'. Lentiviral particles were produced as previously described [32]. The MCF7 cells were infected with lentivirus in the presence of $8 \mu \mathrm{g} / \mathrm{ml}$ polybrene (Sigma-Aldrich). Approximately $48 \mathrm{~h}$ post-infection, the cells were selected by treating with $1 \mu \mathrm{g} / \mathrm{ml}$ puromycin (InvivoGen, San Diego, CA) for 3 days.

\section{Genomic PCR, T7 endonuclease assay, and sequencing}

We extracted genomic DNA from wildtype and Cas9/ sgRNA transduced and puromycin selected MCF7 cells using the Pure Link Genomic DNA Mini-kit (Invitrogen). Primers were designed to amplify a $\sim 700$ base fragment surrounding the sgRNA cleavage site. miR-23b primers: forward 5'- CTCCCCAGCATCTTCGATCC-3' and reverse 5' - GAGGTCATCGCTGGGCATAA-3'; and miR27b primers: forward $5^{\prime}$ - CAGGTGCATCTCGTAGC TCT-3' and reverse 5' ${ }^{\prime}$ - TTTGCTCAAGGGCTCGA CTC-3'. The genomic loci of interest were amplified by PCR using Phusion High-Fidelity DNA Polymerase (Thermo-Scientific). We applied the T7 Endonuclease assay to evaluate the gene editing efficiency [39]. Single cell clones of each transduced cell line were expanded. The PCR amplicons of each clone were inserted and replicated in the $\mathrm{pCR}^{\mathrm{Tm}} 4-\mathrm{TOPO}^{\circ} \mathrm{TA}$ vector (Thermo-Fisher), and sent for Sanger sequencing using the T7 primer (5' TAATACGACTCACTATAGGG 3').

\section{RNA extraction and real-time PCR}

Total RNA was prepared with TRIzol reagent (Invitrogen/Thermo-Fisher) followed by the PureLink RNA Mini Kit (Invitrogen). RNA concentration was determined on the NanoDrop ND-100 Spectrophotometer (NanoDrop Technologies). For miRNA expression analysis, cDNA was synthesized from $100 \mathrm{ng}$ of total RNA using the Quanta qScript microRNA cDNA Synthesis Kit (Quanta Biosciences). The cDNA was combined with 2X iTaq Universal SYBR Green Mix (Bio-Rad), $200 \mathrm{nM}$ PerfeCTa Universal PCR primer, and $200 \mathrm{nM}$ PerfeCTa microRNA assay primers for miR-23a-3p, miR-23b-3p, miR-27a-3p, miR-27b-3p, and miR-24b-3p, and the normalization control SNORD44. For mRNA expression analysis, cDNA was synthesized from $500 \mathrm{ng}$ total RNA using the iScript cDNA Synthesis Kit (Bio-Rad). The cDNA was diluted $(1,10)$ and combined with $500 \mathrm{nM}$ of forward and reverse primers. Primer sequences are as follows: AMOTL1, forward 5'-CGAGGGACTGAACTAGCCAT-3' and reverse 5'-AGGGGACCCTTTCACC G-3'; VHL, forward 5'-ACGGACAGCCTATTTTTGC CA-3' and reverse $5^{\prime}$-TCTTCGTAGAGCGACCTGAC3'; ST14, forward 5'-CACCCAACATTGACTGCACAT$3^{\prime}$ and reverse 5'-GCAGTATTTCTCCCCGTTGAT-3'; APC, forward 5' -AACGAGCACAGAGGTCATC-3' and reverse 5'-GGCTGTTTCATGGTCCATTCG-3'; and the normalization control 36B4, forward 5 '-ATCAACGGGTACAAACGAGTCCTG-3' and reverse 5' - AAGGCAG ATGGATCAGCCAAGAAG-3'. PCR reactions were run on the Bio-Rad CFX 96 Real-Time PCR (Bio-Rad, Hercules, CA) as described [39]. Relative miRNA expression was assessed using the differences in normalized $\mathrm{Ct}$ $(\Delta \Delta \mathrm{Ct}$ method) after normalization.

\section{MTS cell proliferation assay}

Cells were plated onto a 96-well plate at a density of 12 , 000 cells/well in quadruplicate, and cultured at $37^{\circ} \mathrm{C}$ with $5 \% \mathrm{CO}^{2}$ for $1-3$ days. For each well, the attached cells were incubated in $100 \mu \mathrm{L}$ growth medium supplemented with $20 \mu \mathrm{L}$ CellTiter $96^{\circ}$ AQueous One Solution (Promega, Madison, WI, USA) and incubated for $1 \mathrm{~h}$. The absorbance value at $495 \mathrm{~nm}$ was recorded using a spectrometer.

\section{Wound healing/migration assay}

To assess cell migration $1 \times 10^{6}$ cells were seeded to a 6 well plate in triplicate. When the cells reached at least $90 \%$ confluency a sterile $200 \mu$ micropipette tip was used to make 3-4 separate "wounds" through the cells. Culture medium and any floating cells were removed and replaced with fresh medium. Cells were incubated at $37^{\circ} \mathrm{C}$ with $5 \% \mathrm{CO}_{2}$. Initial wound images and the width of each "wound" were measured at the same position for the next 3 days on an Olympus IX51 inverted microscope using the cellSens Standard imaging software (Olympus, Tokyo, Japan).

\section{Soft agar colony formation assay}

The soft agar colony formation assay was used to assess the anchorage-independent growth of the miRNA knockout cells. For the bottom layer, sterilized 1\% agarose LE was diluted 1:1 in complete cell culture medium and $1 \mathrm{ml}$ was transferred to each well of a 6-well plate, for a final agarose concentration of $0.5 \%$. The agarose was allowed to solidify at room temperature for $30 \mathrm{~min}$. To prepare the upper layer, cells were harvested and diluted to a density of 10,000 cells/ $0.5 \mathrm{~mL}$ of complete medium. To this cell suspension, $0.5 \mathrm{ml}$ of sterilized $0.6 \%$ agarose was added, and $1 \mathrm{ml}$ of the mixture was transferred to each well, for a final agarose concentration of $0.3 \%$. The cell/agarose mixture was allowed to solidify at room temperature for $30 \mathrm{~min}$. The cells were incubated at $37{ }^{\circ} \mathrm{C}$ with $5 \% \mathrm{CO}_{2}$ for $20-30$ days. Every 2-3 days $0.2 \mathrm{ml}$ of complete medium was added to each well to prevent drying. Colonies were stained with $0.005 \%$ crystal violet in $25 \%$ methanol for $1 \mathrm{~h}$. Images and colony counts were obtained using the Optronix Gel Count (Oxford Optronix Ltd.). 


\section{Clonogenic assay}

To assess the ability of the miRNA knockout cells to proliferate indefinitely, the cells were seeded at a density of 500 cells/well in a 6-well plate and incubated at $37^{\circ} \mathrm{C}$ with $5 \% \mathrm{CO}_{2}$ for 10-14 days. The culture media was refreshed every 3-4 days. Cells were stained with $0.5 \%$ crystal violet in $25 \%$ methanol for $15 \mathrm{~min}$. The stained cells were washed 3 times with tap water and allowed to dry on the bench. Images and colony counts were obtained using the Optronix Gel Count (Oxford Optronix Ltd.).

\section{Human breast cancer xenograft mice}

Five-week-old female athymic nude mice (NCRNU-F, Taconic Farms, Inc.) were used for the in vivo study. The animal research protocol was approved by and performed per the policies and guidelines of the University of Oklahoma Health Sciences Center Institutional Animal Care and Use Committee (Institute IACUC Protocol \#100861-14-025-SSH). Mice were kept in a conventional animal facility, with a 12-h on-off light cycle in standard rectangular mouse cages, with 5 mice per cage. Cages were lined with adsorbent bedding and enriched with crinkle paper for nesting. Mice had continuous access to water and food. One week prior to breast cancer cell inoculation the mice were randomly divided into two groups, a standard diet consisting of 0.45\% n-3 fatty acids (PicoLab Rodent Diet-20 \#5053, LabDiet), a fish oil diet consisting of 7.5\% n-3 fatty acids (Envigo TD.110647), and fed with the assigned diet during the whole duration of the experiment. Three days before cell inoculation the mice were supplemented with $17 \beta$ estradiol in the form of a $0.36 \mathrm{mg} /$ pellet, 60-day release (SE-121, Innovative Research of America) implanted subcutaneously in the intrascapular region. The MCF7 miR-23b and miR-27b knockout or control cells were inoculated at a density of $5 \times 10^{6}$ in $100 \mu \mathrm{l}$ PBS containing $5 \mu \mathrm{g} / \mathrm{ml}$ Matrigel (BD Biosciences) subcutaneously into the mammary fat pad $(n=6$ mice per group). Mouse weight and tumor size measurements were initiated 7 days post inoculation and monitored three times per week. Tumor measurements were taken using digital calipers. Tumor volume (v) was measured using the following formula: $\mathrm{v}=\left(\mathrm{l} \times \mathrm{w}^{2}\right) \times 0.5$, where $\mathrm{l}$ is the length and $w$ is the width of the tumor measured in $\mathrm{mm}$. Tumor weight (W, in grams) was estimated by the following formula: $\mathrm{W}(\mathrm{g})=\mathrm{v}\left(\mathrm{mm}^{3}\right) \times 0.001$. When the estimated tumor weight reached or exceeded $10 \%$ of the body weight, the mouse was euthanized by $\mathrm{CO}_{2}$ asphyxiation. No adverse events were observed. Tumor tissues were dissected, fixed in $10 \%$ buffered formalin phosphate, followed by paraffin embedding, sectioning, hematoxylin, and eosin (H/E) staining. Survival analysis was conducted in GraphPad Prism (version 7). The date of death was entered for mice within each group over the entire study period. The survival curves were compared using the Log-rank test (Mantel-Cox).

\section{Immunohistochemical staining of CD31 and Ki67}

Tumor tissues were dissected from mice upon sacrifice and fixed in a $10 \%$ neutral buffered-Formalin solution and embedded in paraffin wax. FFPE tissues were sectioned $(4 \mu \mathrm{m})$, mounted on positively charged slides and dried overnight at room temperature. Slides were then incubated at $60^{\circ} \mathrm{C}$ for $45 \mathrm{~min}$, followed by deparaffinization, and rehydration in an automated Multistainer (Leica ST5020). Slides were transferred to the Leica Bond- $\mathrm{III}^{\mathrm{mi}}$ and treated for target retrieval at $100^{\circ} \mathrm{C}$ for $20 \mathrm{~min}$ in a retrieval solution (at $\mathrm{pH} 6.0$ or $\mathrm{pH} 9.0$ ). Endogenous peroxidase enzyme was blocked with a peroxidase-blocking reagent, followed by primary antibody incubation for $60 \mathrm{~min}$. Tumor sections were evaluated by immunohistochemistry for neovascularization using an antibody against CD31 (1:50; ab28364, Abcam) and for proliferation using an antibody against Ki67 (1: 800; ab15580, Abcam). Post-primary IgG-linker and/or Poly-HRP IgG secondary antibody reagents were used for detection with 3, 3'-diaminobenzidine tetrahydrochloride $(\mathrm{DAB})$ as chromogen. Slides were counterstained with hematoxylin. Stained slides were dehydrated (Leica ST5020) and mounted (Leica MM24). Primary antibody specific positive and negative controls (omission of primary antibody) were stained in parallel. Stained slides were scanned and analyzed using the Aperio CS2 scanner and ImageScope software v12 (Leica Biosystems). To analyze nuclear Ki67 staining the Nuclear Staining Algorithm was used to calculate the percent of nuclei positive among 4 randomly chosen areas within each xenograft tumor section. The Microvessel Analysis Algorithm was used to quantify the number of CD31 positive microvessels present on each tumor xenograft section.

\section{Results \\ CRISPR/Cas9 knockout of miR-23b and miR-27b in MCF7 cells}

To investigate the functional role of miR-23b and miR$27 \mathrm{~b}$ in breast cancer, we performed a loss-of-function study using the CRISPR/Cas9 system. We constructed CRISPR/Cas9 vectors containing 2 different sgRNAs designed to target the MIR23B and the MIR27B gene locus on chromosome 9. The sgRNAs were designed using the DNA 2.0 sgRNA design tool with the DNA sequence corresponding to the stem-loop sequence of the miRNA. Insertion of the sgRNAs into the lenti-CRISPR/ Cas9 v2 vector was confirmed by DNA sequencing. MCF7 cells were transduced with the prepared lentivirus containing either empty vector or targeting sgRNAs. The T7 Endonuclease assay was used to assess the gene 
editing efficiency and single cell clones of each transduced cell line were plated and expanded. The genetic region surrounding the sgRNA target site of each selected clone was PCR amplified and ligated into the pCR4-TOPO TA vector and sequenced for mutation pattern determination. Indels were confirmed in single clones from each of the targeting sgRNAs (two per sgRNA), with genetic deletions observed in both miR23b-1 and miR-23b-2 clones and deletions in both the miR-27b-1 clones, while $1 \mathrm{bp}$ and $8 \mathrm{bp}$ insertions were observed in the miR-27b-2 clones (Fig. 1a). Knockout of miR-23b and miR-27b was confirmed by qRT-PCR, thus demonstrating the high efficiency of the CRISPR/Cas9 system (Fig. 1b). Expression levels of the non-targeted homologs, miR-23a and miR-27a, were also measured in the knockout cells. As shown in Fig. 1c, while a slight increase in miR-27a was observed in the miR-27b-1 \#10 clone, no significant changes in miR-23a or mir-27a expression were observed in any of the clones. Because miR-23b and miR-27b are located in a cluster on chromosome 9 at a distance of only $141 \mathrm{bp}$, we also assessed the expression of the non-targeted miRNA and the neighboring miR-24b in each of the clones. As shown in Fig. 1d, e the expression of the non-targeted miRNA was modestly changed in 3 out of the 4 targeted clones relative to the empty vector control. While, miR$24 \mathrm{~b}$ was modestly increased in the miR-23b clones, and decreased in the miR-27b clones (Fig. 1f). This suggests that the presence of the indel in the adjacent miRNA precursor sequence was minimally disruptive to the normal expression of the other clustered miRNAs, relative to the highly significant repression of the targeted miRNA.

To assess the effects of miRNA knockdown, we additionally evaluated the expression of previously verified miR-23b/27b target genes. Angiomotin like 1 (AMOTL1), that promotes tube formation and migration of endothelial cells and regulates tight junctions, cell polarity, and epithelial-mesenchymal transition in epithelial cells [41], and Von Hippel-Lindau (VHL), a

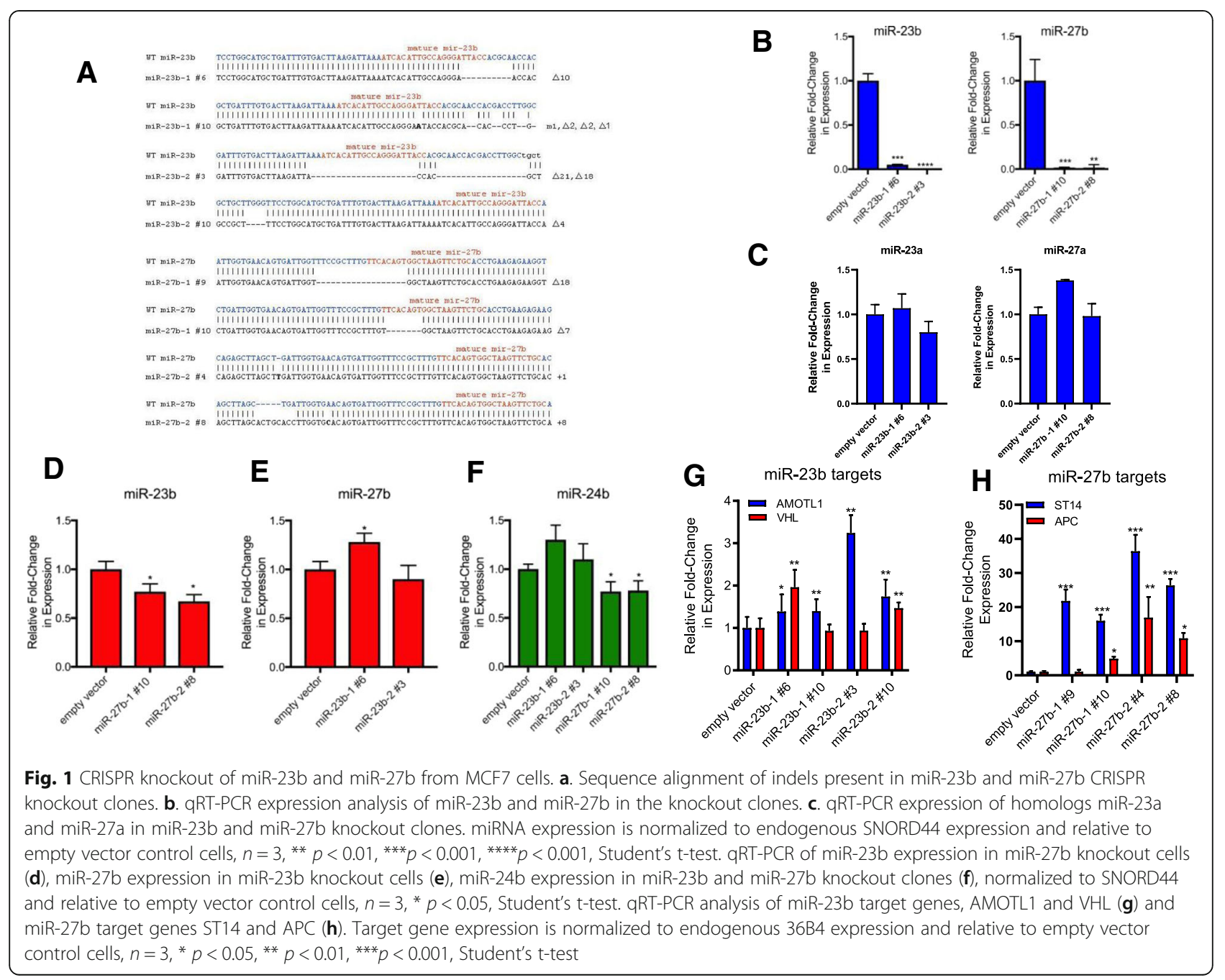




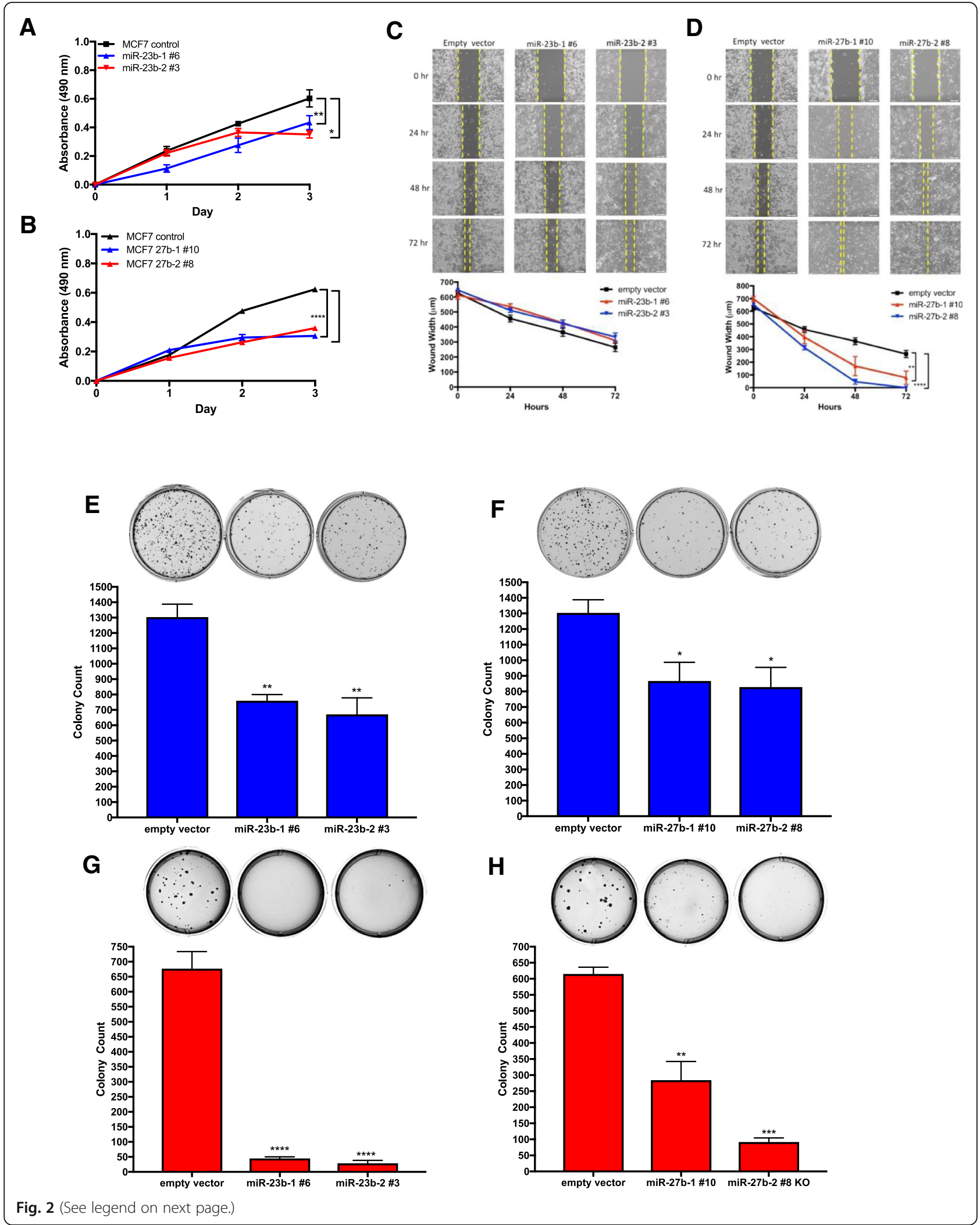


(See figure on previous page.)

Fig. 2 miR-23b and miR-27b knockout cell growth and migration characteristics. Cellular growth curve of miR-23b (a) and miR-27b (b) knockout clones. Cells were plated in quadruplicate and proliferation was measured by MTS assay, ${ }^{*} p<0.05,{ }^{* *} p<0.01,{ }^{* * *} p<0.0001$, Student's t-test. Cell migration of miR-23b (c) and miR-27b (d) knockout cells was measured compared to empty vector control cells by the wound healing assay. A wound was made in a monolayer of cells and migration into the wound was measured every $24 \mathrm{~h}$ for a total of $72 \mathrm{~h}$. Representative images (20X magnification) of the wound were captured (upper) and the rate of wound closure was calculated (lower); $n=6$ per time point, ${ }^{* *} p<0.01$, **** $p<0.0001$, Two-way ANOVA. Clonogenic assay of miR-23b (e) and miR-27b (f) knockout cells compared to empty vector control cells. Soft-agar assay of miR-23b (g) and miR-27b (h) knockout cells compared to empty vector control cells. Representative images of colony growth and colony counts of the average of 2 experiments performed in triplicate; ${ }^{*} p<0.05,{ }^{* *} p<0.01$, ${ }^{* *} p<0.001$, ${ }^{* * *} p<0.0001$, One-way ANOVA, Dunnett's post-test

tumor suppressor involved in the ubiquitination and degradation of hypoxia-inducible-factor, are verified miR-23b target genes [42, 43]. Suppression of tumorigenicity 14 (ST14), also known as matriptase, is an epithelial cell-specific membrane-anchored serine protease found to reduce cell proliferation and invasion of cancer cells [23], and adenomatous polyposis coli (APC), a tumor suppressor protein that acts as an antagonist of the Wnt signaling pathway [44], are both validated miR$27 \mathrm{~b}$ targets. In the miR-23b knockout cells, we observed a significant increase in expression of AMOTL1 in all clones tested. A significant increase in VHL levels was observed in clone miR-23b-1 \#6 and miR-23b-2 \#10, whereas no significant change in VHL levels was observed in 2 of the 4 clones tested (Fig. 1g). Meanwhile, in the miR-27b knockout cells, we observed a significant increase of ST14 in all clones tested, while APC expression levels were significantly elevated in 3 out of the 4 clones tested (Fig. 1h). These data indicate that knockout of miR-23b/27b affects the expression of specific target genes in breast cancer cells.

\section{miR-23b/27b depletion alters breast cancer cell behavior in vitro}

To examine the growth characteristics of the knockout cells we conducted an MTS growth assay. The rate of proliferation of both $\mathrm{miR}-23 \mathrm{~b} / 27 \mathrm{~b}$ depleted cells was significantly reduced compared to the control cells (Fig. 2a, b). In addition to a reduced proliferation rate, we also assessed the migration/wound-healing rate of the knockout cells. No significant change in the rate of migration was observed in the miR-23b knockout cells compared to control cells (Fig. 2c) over a $72 \mathrm{~h}$ period. However, the miR-27b depleted clones migrated to close the artificial wound at a significantly faster rate compared to the control cells (Fig. 2d).

Using the clonogenic assay, we sought to assess whether the knockout cells had retained their ability to produce colonies. The knockout and control cells were plated at a low density, cultured for two weeks, stained, and counted. Both the miR-23b and miR-27b knockout clones generated significantly fewer colonies compared to control cells (Fig. 2e, f), thus demonstrating a reduced replicative ability in the absence of $\mathrm{miR}-23 \mathrm{~b} / 27 \mathrm{~b}$. We also assessed the ability of the depleted cells to grow independently of a solid surface, a known hallmark of carcinogenesis, using the soft-agar colony forming assay. Cells were suspended in agar and cultured for 20 to 30 days. The depletion of miR-23b/27b significantly attenuated anchorage-independent growth (Fig. 2g, h). These results indicated that genetic depletion of miR-23b/27b inhibits cell proliferation, colony formation, and anchorage-independent growth in vitro. However, cell migration was enhanced in miR-27b depleted cells, and unchanged in miR-23b depleted cells, indicating a functional difference between miR-23b and miR-27b, and that miR-27b plays a role in reducing cell mobility.

\section{miR-23b/27b knockout reduces tumor growth in vivo}

To evaluate the in vivo effects of genetic depletion of $\mathrm{miR}-23 \mathrm{~b} / 27 \mathrm{~b}$ on tumorigenesis, we subcutaneously implanted the CRISPR empty vector control cells, miR$23 \mathrm{~b}$ and miR-27b knockout cells ( $n=6$ mice per group, 3 groups total) into the mammary fat pad of randomly selected nude mice and monitored tumor growth for up to 6 weeks. Due to the consistent upregulation of known target genes, the miR-23b-1 \#6 and the miR-27b-1 \#10 clones were selected for these experiments. Due to the rapid rate of empty vector control cell xenograft growth, this group of mice was sacrificed at 3 weeks postinjection. The growth of $\mathrm{miR}-23 \mathrm{~b} / \mathrm{miR}-27 \mathrm{~b}$ knockout xenografts was significantly reduced compared to empty vector control cells at the third week of growth as determined by tumor volumes, with the miR-23b knockout being more dramatic (Fig. 3a). Mouse survival was significantly increased in both miR-23b and miR-27b knockout xenografts (Fig. 3b). The tumor xenograft growth inhibitory effect was confirmed by immunohistochemical staining of tumor cell proliferation at the endpoint using the cell cycle marker, Ki-67 (Fig. 3c, d). These data indicate that miR-23b and miR-27b function primarily as oncogenes in breast cancer cells, and their genetic depletion significantly reduces tumor growth in vivo, with miR-23b deletion being more effective.

We previously reported that the anti-cancer and antiangiogenesis agent docosahexaenoic acid, the primary long-chain n-3 fatty acid found in fish-oil, induced secretion of miR-23b and miR-27b via exosomes that may 

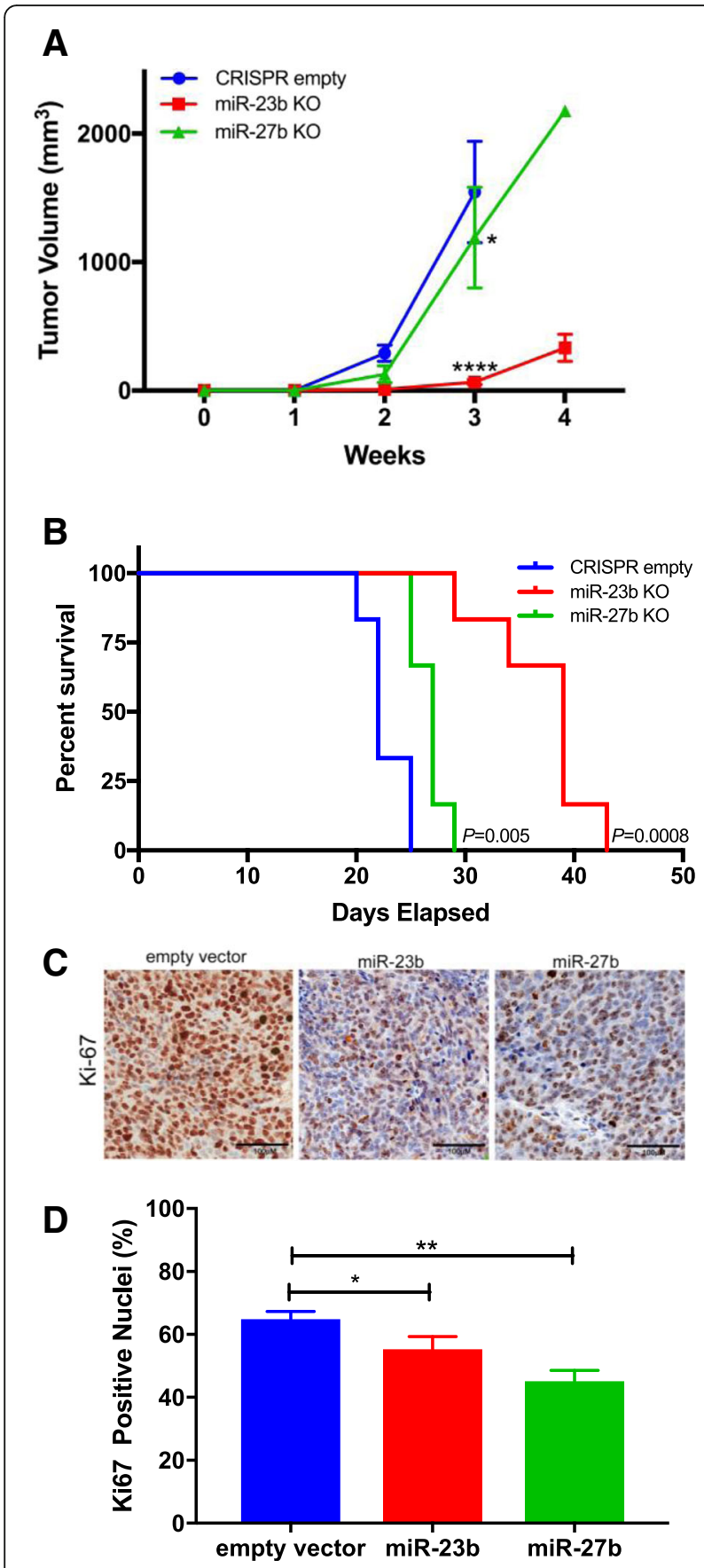

Fig. 3 miR-23b and miR-27b knockout cell orthotopic tumor xenograft growth. a. Tumor xenograft growth curve, $n=6$ mice per group, Two-way ANOVA, ${ }^{*} p<0.05,{ }^{* * * *} p<0.0001$. b. Survival analysis of empty vector, miR-23b, and miR-27b, knockout xenograft mice fed a standard diet, $n=6$ mice per group. Survival curves were compared using the Log-rank (Mantel-Cox) test in GraphPad Prism. Representative images (c) and quantification (d) of Ki-67 immunohistochemistry staining of xenograft tumor tissue, black bar $=100 \mu \mathrm{m} ; n=9$ per group, ${ }^{*} p<0.05,{ }^{* *} p<0.01$, Student's t-test contribute to the anti-angiogenesis activity of DHA [32]. Therefore, we evaluated the effect of a fish-oil diet on growth and angiogenesis of MCF7 miR-23b/27b knockout xenografts. Mice were randomly selected to receive either a standard mouse diet or a fish oil-based diet consisting of $75 \mathrm{~g} / \mathrm{kg}$ of fish oil, as we previously reported [45]. CRISPR empty vector control, miR-23b, and miR-27b knockout cells were subcutaneously implanted into the mammary fat pad ( $n=6$ mice per group, 3 groups total) and tumor growth and volume were monitored up to 8 weeks. The rate of growth of the empty vector and miR-23b xenografts was significantly attenuated in the mice fed a fish-oil diet, whereas the miR-27b knockout xenograft was unchanged (Fig. 4a). Survival of control and miR-23b knockout xenograft mice was significantly increased in mice fed a fish oil diet compared to those fed a standard diet, whereas the survival of miR-27b xenograft mice fed a fish oil diet was unchanged from those provided a standard diet (Fig. 4b). Immunohistochemical staining using antibodies against CD31 showed that the density of microvessels in the xenograft tumors was significantly reduced in both miR23b and miR-27b xenograft mice fed a fish-oil diet (Fig. 4c, d). These results suggest that the antitumorigenic activity of DHA, provided by the fish oil diet, is abolished by miR-27b knockout in the tumor cells, while the anti-angiogenic activity of DHA is unaffected by miR-23b or miR-27b knockout.

\section{Discussion}

Although previous studies have successfully used miRNA antagonists to reduce miRNA expression of miR-23b and miR-27b, the efficiency of knockdown is often incomplete and temporary [18]. The CRISPR/Cas9 system for gene editing and genomic depletion is significantly more efficient, producing permanent gene knockout [46]. While the majority of studies using the CRISPR/Cas9 system have focused on the depletion of protein-coding genes, very few studies have targeted non-coding RNA regions, including miRNAs for genomic depletion [47]. In this study, we utilized sgRNAs designed to target within the stem-loop sequence of miR-23b and miR-27b. The knockout efficiency was greater than $90 \%$ in all clones tested. The functional significance of $\mathrm{miR}-23 \mathrm{~b} / 27 \mathrm{~b}$ depletion was examined in MCF7 breast cancer cells. Depletion of miR-23b and miR-27b allowed the re-expression of several of their target genes that are known to be involved in various mechanisms of cancer progression, indicating that expression of miR-23b and miR-27b target genes are affected by the loss of each individual miRNA.

We further characterized the growth and migratory characteristics of the miR-23b and miR-27b knockout cells in vitro. The knockdown approach, suggests that 


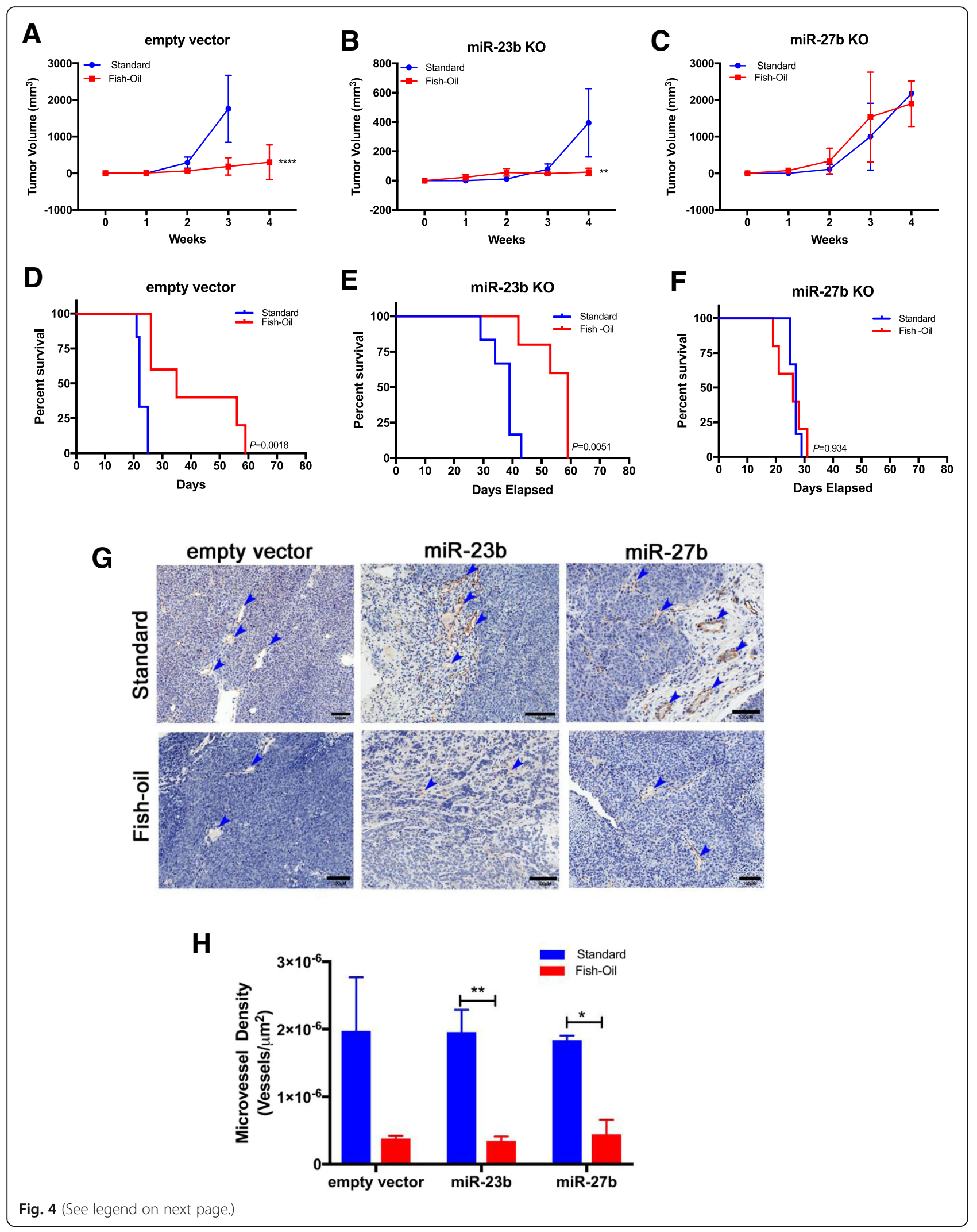


(See figure on previous page.)

Fig. 4 Effect of fish oil diet on the growth of miR-23b and miR-27b knockout cell orthotopic tumor xenografts. Tumor xenograft growth curve of empty vector (a), miR-23b (b) and miR-27b (c) knockout xenograft mice fed a fish-oil based diet versus a standard diet; $\mathrm{n}=6$ mice per group, ** $p<0.01,{ }^{* * * *} p<0.0001$, Two-way ANOVA. Survival analysis of empty vector (d), miR-23b (e) and miR-27b (f) knockout xenograft mice fed a fish-oil based diet versus a standard diet. Survival curves were compared using the Log-rank (Mantel-Cox) test in GraphPad Prism g. Representative images and $\mathbf{h}$. quantitation of immunohistochemistry staining CD31 positive microvessels in xenograft tumor specimens, black bar $=100 \mu \mathrm{m} ; n=3$ per group, ${ }^{*} p<0.05,{ }^{* *} p<0.01$, Student's t-test. Blue arrows indicate representative regions of CD31 positive microvessels

the complete elimination of expression of miR-23b and miR-27b significantly decreased the cell growth and colony forming ability of these cells compared to the empty vector control cells, strongly indicating the oncogenic nature of these two miRNAs. However, the rate of cell migration was significantly reduced in miR-27b knockout cells, while miR-23b knockout cells migratory behavior was unchanged, indicating a functional difference between these two miRNAs. When miR-23b and miR27b knockout cell growth was measured in vivo, both tumor xenografts grew at a slower rate, with miR-23b knockout xenografts being more dramatic, compared to empty vector control, consistent with in vitro observations. However, when mice were provided a fish-oil based diet, the rate of growth of empty vector control and miR-23b knockout tumor xenografts was both significantly reduced; while the growth of miR-27b knockout xenografts was similar to that in mice fed a standard diet. Tumor neovascularization as determined by the tumor xenograft microvessel density was significantly reduced in the miR-23b and miR-27b knockout xenografts from mice fed a fish-oil based diet, regardless of the cell lines injected, which further supports the anti-angiogenic role of fish-oil and indicates that miR23b and miR-27b are not involved in fish-oil's antiangiogenic activity. Some of the limitations of this study include that MCF7 cells are non-metastatic, and therefore metastasis could not be observed or evaluated in this model system; and the fish oil diet contains different n-3 polyunsaturated fatty acids and from a mechanistic point of view, a diet with DHA specific addition would be more supportive of our conclusion.

Because miR-23b and miR-27b belong to the same miRNA cluster, it is theoretically difficult to selectively and permanently eliminate the expression of one miRNA without affecting the other. In the present study, we were able to eliminate one miRNA at once with minimal effect on the other clustered miRNAs using the CRISPR/Cas9 gene editing technology, thus allowing us to dissect the functional difference of these two miRNAs. An interesting finding of the present study is that, although miR-23b and miR-27b both act primarily as oncogenic miRNAs (Figs. 2a, b, d-h and 3), miR-27b possesses context-dependent tumor suppressor activity, as evidenced by its inhibitory effect on cell migration (Fig. 2d) and the diminished effect on fish oil-induced suppression of tumor growth (Fig. 4c, f). This seems to be consistent with our in vivo data strongly indicating that miR-23b is a more potent oncogenic miRNA than miR-27b (Fig. 3a, b). Whereas the mechanisms of miR27b's context-dependent activity remain to be further elucidated, these observations may partially explain previous confusion over the dichotomous role of this miRNA in cancer progression.

\section{Conclusions}

Taken together, our findings show that genetic depletion of miR-23b and miR-27b results in suppression of tumor growth, indicating that miR-23b and miR-27b are indeed oncogenic miRNAs in breast cancer. Our work suggests that the dysregulated expression of $m i R-23 b / 27 b$ in breast cancer is a significant driving force behind breast cancer progression and therapeutic targeting of these miRNAs is a plausible strategy for breast cancer management. This study also demonstrates that miR-27b is a less potent oncogenic miRNA that also possesses context-dependent tumor suppressor activity.

\section{Abbreviations}

CRISPR: Clustered regularly interspaced short palindromic repeats; DHA: Docosahexaenoic acid; miRNA: microRNA; sgRNA: Single-guide RNA; UTR: Untranslated region

\section{Acknowledgements}

We thank the Stephenson Cancer Center at the University of Oklahoma Health Sciences Center, Oklahoma City, OK and an Institutional Development Award (IDeA) from the National Institute of General Medical Sciences of the National Institutes of Health under grant number P20 GM103639 for the use of Tissue Pathology Core, which provided immunohistochemistry and image analysis services.

\section{Authors' contributions}

$\mathrm{BNH}$ designed the study, carried out and/or supervised the experiments and drafted the manuscript. AC and CLC assisted with in vitro cellular assays. YFX assisted with in vivo animal studies. RZ and KMF assisted with histopathology and immunohistochemical analysis of mouse tissues from in vivo studies. WQD conceived of the study and participated in its design and coordination and helped to draft the manuscript. All authors read and approved the final manuscript.

\section{Funding}

This study was supported in part by grants from the Oklahoma Center for the Advancement of Science and Technology (HR14-147, HR17-052); the National Institute of General Medical Sciences of the National Institutes of Health (U54GM104938); and Presbyterian Health Foundation. These funds provided support for personnel, lab supplies, and equipment operations.

\section{Availability of data and materials}

The datasets used and/or analyzed during the current study are available from the corresponding author on reasonable request. 


\section{Ethics approval and consent to participate}

Athymic nude mice (Foxn1nu) were used for in vivo evaluation of miR-23b and miR-27b function in cancer progression per the Institute Animal Care and Use Committee procedures and guidelines (University of Oklahoma Health Sciences Center IACUC Protocol\#: 100861-14-025-SSH). The use of the MCF7 cell line for research was approved by the Institutional Review Board at the University of Oklahoma Health Sciences Center (IRB\#: 2424).

\section{Consent for publication}

Not applicable.

\section{Competing interests}

The authors declare that they have no competing interests.

\section{Received: 29 August 2018 Accepted: 17 June 2019}

Published online: 28 June 2019

\section{References}

1. Lim LP, Lau NC, Garrett-Engele P, Grimson A, Schelter JM, Castle J, Bartel DP, Linsley PS, Johnson JM. Microarray analysis shows that some microRNAs downregulate large numbers of target mRNAs. Nature. 2005;433(7027):769-73.

2. Taft RJ, Pang KC, Mercer TR, Dinger M, Mattick JS. Non-coding RNAs: regulators of disease. J Pathol. 2010;220(2):126-39.

3. Griffiths-Jones S, Grocock RJ, van Dongen S, Bateman A, Enright AJ. miRBase: microRNA sequences, targets and gene nomenclature. Nucleic Acids Res. 2006;34(Database issue):D140-4.

4. Panwar B, Omenn GS, Guan Y. miRmine: a database of human miRNA expression profiles. Bioinformatics. 2017;33(10):1554-60

5. Chou CH, Chang NW, Shrestha S, Hsu SD, Lin YL, Lee WH, Yang CD, Hong HC, Wei TY, Tu SJ, et al. miRTarBase 2016: updates to the experimentally validated miRNA-target interactions database. Nucleic Acids Res. 2016; 44(D1):D239-47.

6. He HC, Zhu JG, Chen XB, Chen SM, Han ZD, Dai QS, Ling XH, Fu X, Lin ZY, Deng $Y H$, et al. MicroRNA-23b downregulates peroxiredoxin III in human prostate cancer. FEBS Lett. 2012:586(16):2451-8.

7. Majid S, Dar AA, Saini S, Arora S, Shahryari V, Zaman MS, Chang I, Yamamura S, Tanaka Y, Deng G, et al. miR-23b represses proto-oncogene Src kinase and functions as methylation-silenced tumor suppressor with diagnostic and prognostic significance in prostate cancer. Cancer Res. 2012;72(24): 6435-46.

8. Majid S, Dar AA, Saini S, Deng G, Chang I, Greene K, Tanaka Y, Dahiya R, Yamamura S. MicroRNA-23b functions as a tumor suppressor by regulating Zeb1 in bladder cancer. PLoS One. 2013;8(7):e67686.

9. Chiyomaru T, Seki N, Inoguchi S, Ishihara T, Mataki H, Matsushita R, Goto Y, Nishikawa R, Tatarano S, Itesako T, et al. Dual regulation of receptor tyrosine kinase genes EGFR and c-met by the tumor-suppressive microRNA-23b/27b cluster in bladder cancer. Int J Oncol. 2015;46(2):487-96.

10. Pellegrino L, Stebbing J, Braga VM, Frampton AE, Jacob J, Buluwela L, Jiao LR, Periyasamy M, Madsen CD, Caley MP, et al. miR-23b regulates cytoskeletal remodeling, motility and metastasis by directly targeting multiple transcripts. Nucleic Acids Res. 2013;41(10):5400-12.

11. Ono M, Kosaka N, Tominaga N, Yoshioka Y, Takeshita F, Takahashi RU, Yoshida M, Tsuda $\mathrm{H}$, Tamura K, Ochiya T. Exosomes from bone marrow mesenchymal stem cells contain a microRNA that promotes dormancy in metastatic breast cancer cells. Sci Signal. 2014;7(332):ra63.

12. An $Y$, Zhang $Z$, Shang $Y$, Jiang $X$, Dong J, Yu P, Nie $Y$, Zhao Q. miR-23b-3p regulates the chemoresistance of gastric cancer cells by targeting ATG12 and HMGB2. Cell Death Dis. 2015;6:e1766.

13. Huang TT, Ping $\mathrm{YH}$, Wang $\mathrm{AM}$, Ke CC, Fang WL, Huang $\mathrm{KH}$, Lee HC, Chi CW, Yeh TS. The reciprocal regulation loop of Notch2 pathway and miR-23b in controlling gastric carcinogenesis. Oncotarget. 2015;6(20):18012-26.

14. Zhang H, Hao Y, Yang J, Zhou Y, Li J, Yin S, Sun C, Ma M, Huang Y, Xi JJ. Genome-wide functional screening of miR-23b as a pleiotropic modulator suppressing cancer metastasis. Nat Commun. 2011;2:554.

15. Geng J, Luo H, Pu Y, Zhou Z, Wu X, Xu W, Yang Z. Methylation mediated silencing of miR-23b expression and its role in glioma stem cells. Neurosci Lett. 2012;528(2):185-9.

16. Vaksman O, Trope C, Davidson B, Reich R. Exosome-derived miRNAs and ovarian carcinoma progression. Carcinogenesis. 2014;35(9):2113-20.
17. Tian L, Fang $Y X, X u e ~ J L, C$ Chen JZ. Four microRNAs promote prostate cell proliferation with regulation of PTEN and its downstream signals in vitro. PLoS One. 2013;8(9):e75885.

18. Jin L, Wessely O, Marcusson EG, Ivan C, Calin GA, Alahari SK. Prooncogenic factors miR-23b and miR-27b are regulated by Her2/Neu, EGF, and TNFalpha in breast cancer. Cancer Res. 2013;73(9):2884-96.

19. Qi P, Xu MD, Shen XH, Ni SJ, Huang D, Tan C, Weng WW, Sheng WQ, Zhou $X Y$, Du X. Reciprocal repression between TUSC7 and miR-23b in gastric cancer. Int J Cancer. 2015;137(6):1269-78.

20. Chen L, Zhang K, Shi Z, Zhang A, Jia Z, Wang G, Pu P, Kang C, Han L. A lentivirus-mediated miR-23b sponge diminishes the malignant phenotype of glioma cells in vitro and in vivo. Oncol Rep. 2014;31(4):1573-80.

21. Chen L, Li H, Han L, Zhang K, Wang G, Wang Y, Liu Y, Zheng Y, Jiang T, Pu $P$, et al. Expression and function of miR-27b in human glioma. Oncol Rep. 2011;26(6):1617-21.

22. Liu F, Zhang S, Zhao Z, Mao X, Huang J, Wu Z, Zheng L, Wang Q. MicroRNA-27b up-regulated by human papillomavirus 16 E7 promotes proliferation and suppresses apoptosis by targeting polo-like kinase 2 in cervical cancer. Oncotarget. 2016;7(15):19666-79.

23. Wang Y, Rathinam R, Walch A, Alahari SK. ST14 (suppression of tumorigenicity 14) gene is a target for miR-27b, and the inhibitory effect of ST14 on cell growth is independent of miR-27b regulation. J Biol Chem. 2009;284(34):23094-106

24. Jiang J, Lv X, Fan L, Huang G, Zhan Y, Wang M, Lu H. MicroRNA-27b suppresses growth and invasion of NSCLC cells by targeting Sp1. Tumour Biol. 2014;35(10):10019-23.

25. Goto Y, Kojima S, Nishikawa R, Enokida H, Chiyomaru T, Kinoshita T, Nakagawa M, Naya Y, Ichikawa T, Seki N. The microRNA-23b/27b/24-1 cluster is a disease progression marker and tumor suppressor in prostate cancer. Oncotarget. 2014;5(17):7748-59.

26. Ye J, Wu X, Wu D, Wu P, Ni C, Zhang Z, Chen Z, Qiu F, Xu J, Huang J. miRNA-27b targets vascular endothelial growth factor $C$ to inhibit tumor progression and angiogenesis in colorectal cancer. PLoS One. 2013;8(4):e60687.

27. Tao J, Zhi X, Zhang X, Fu M, Huang H, Fan Y, Guan W, Zou C. miR-27b-3p suppresses cell proliferation through targeting receptor tyrosine kinase like orphan receptor 1 in gastric cancer. J Exp Clin Cancer Res. 2015;34:139.

28. Lin X, Chen L, Yao Y, Zhao R, Cui X, Chen J, Hou K, Zhang M, Su F, Chen J, et al. CCL18-mediated down-regulation of miR98 and miR27b promotes breast cancer metastasis. Oncotarget. 2015;6(24):20485-99.

29. Zhou Q, Gallagher R, Ufret-Vincenty R, Li X, Olson EN, Wang S. Regulation of angiogenesis and choroidal neovascularization by members of microRNA23 27 24 clusters. Proc Natl Acad Sci U S A. 2011;108(20):8287-92.

30. Urbich C, Kaluza D, Fromel T, Knau A, Bennewitz K, Boon RA, Bonauer A, Doebele C, Boeckel JN, Hergenreider E, et al. MicroRNA-27a/b controls endothelial cell repulsion and angiogenesis by targeting semaphorin 6A. Blood. 2012:119(6):1607-16.

31. Wang KC, Garmire LX, Young A, Nguyen P, Trinh A, Subramaniam S, Wang $\mathrm{N}$, Shyy JY, Li YS, Chien S. Role of microRNA-23b in flow-regulation of Rb phosphorylation and endothelial cell growth. Proc Natl Acad Sci U S A. 2010;107(7):3234-9.

32. Hannafon BN, Carpenter KJ, Berry WL, Janknecht R, Dooley WC, Ding WQ. Exosome-mediated microRNA signaling from breast cancer cells is altered by the anti-angiogenesis agent docosahexaenoic acid (DHA). Mol Cancer. 2015;14:133.

33. Liu HT, Xing AY, Chen X, Ma RR, Wang YW, Shi DB, Zhang H, Li P, Chen HF, Li YH, et al. MicroRNA-27b, microRNA-101 and microRNA-128 inhibit angiogenesis by down-regulating vascular endothelial growth factor $C$ expression in gastric cancers. Oncotarget. 2015;6(35):37458-70.

34. Ell B, Qiu Q, Wei Y, Mercatali L, Ibrahim T, Amadori D, Kang Y. The microRNA-23b/27b/24 cluster promotes breast cancer lung metastasis by targeting metastasis-suppressive gene prosaposin. J Biol Chem. 2014; 289(32):21888-95.

35. Zhu J, Zou Z, Nie P, Kou X, Wu B, Wang S, Song Z, He J. Downregulation of microRNA-27b-3p enhances tamoxifen resistance in breast cancer by increasing NR5A2 and CREB1 expression. Cell Death Dis. 2016;7(11):e2454.

36. Ding L, Ni J, Yang F, Huang L, Deng H, Wu Y, Ding X, Tang J. Promising therapeutic role of miR-27b in tumor. Tumour Biol. 2017:39(3): 1010428317691657

37. Stoll LL, Spector AA. Changes in serum influence the fatty acid composition of established cell lines. In Vitro. 1984;20(9):732-8. 
38. Spector AA, Kiser RE, Denning GM, Koh SW, DeBault LE. Modification of the fatty acid composition of cultured human fibroblasts. J Lipid Res. 1979;20(4): 536-47.

39. Hannafon BN, Gin AL, Xu YF, Bruns M, Calloway CL, Ding WQ. Metastasisassociated protein 1 (MTA1) is transferred by exosomes and contributes to the regulation of hypoxia and estrogen signaling in breast cancer cells. Cell Commun Signal. 2019;17(1):13.

40. Sanjana NE, Shalem O, Zhang F. Improved vectors and genome-wide libraries for CRISPR screening. Nat Methods. 2014;11(8):783-4.

41. Lv M, Shen Y, Yang J, Li S, Wang B, Chen Z, Li P, Liu P, Yang J. Angiomotin family members: oncogenes or tumor suppressors? Int J Biol Sci. 2017;13(6): 772-81.

42. Helwak A, Kudla G, Dudnakova T, Tollervey D. Mapping the human miRNA interactome by CLASH reveals frequent noncanonical binding. Cell. 2013; 153(3):654-65.

43. Chen L, Han L, Zhang K, Shi Z, Zhang J, Zhang A, Wang Y, Song Y, Li Y, Jiang $T$, et al. VHL regulates the effects of miR-23b on glioma survival and invasion via suppression of HIF-1alpha/NEGF and beta-catenin/Tcf-4 signaling. Neuro-Oncology. 2012;14(8):1026-36

44. Zhang Z, Liu S, Shi R, Zhao G. miR-27 promotes human gastric cancer cell metastasis by inducing epithelial-to-mesenchymal transition. Cancer Genet. 2011;204(9):486-91.

45. Jiao Y, Hannafon BN, Zhang RR, Fung KM, Ding WQ. Docosahexaenoic acid and disulfiram act in concert to kill cancer cells: a mutual enhancement of their anticancer actions. Oncotarget. 2017;8(11):17908-20.

46. Chang H, Yi B, Ma R, Zhang X, Zhao H, Xi Y. CRISPR/cas9, a novel genomic tool to knock down microRNA in vitro and in vivo. Sci Rep. 2016;6:22312.

47. Aquino-Jarquin G. Emerging role of CRISPR/Cas9 technology for MicroRNAs editing in cancer research. Cancer Res. 2017;77(24):6812-7.

\section{Publisher's Note}

Springer Nature remains neutral with regard to jurisdictional claims in published maps and institutional affiliations.

Ready to submit your research? Choose BMC and benefit from:

- fast, convenient online submission

- thorough peer review by experienced researchers in your field

- rapid publication on acceptance

- support for research data, including large and complex data types

- gold Open Access which fosters wider collaboration and increased citations

- maximum visibility for your research: over $100 \mathrm{M}$ website views per year

At $\mathrm{BMC}$, research is always in progress.

Learn more biomedcentral.com/submissions 